\title{
No influence of sarcopenia on survival of ovarian cancer patients in a prospective validation study
}

Citation for published version (APA):

Ubachs, J., Koole, S. N., Lahaye, M., Fabris, C., Bruijs, L., van Leeuwen, J. S., Schreuder, H. W. R., Hermans, R. H., de Hingh, I. H., van der Velden, J., Arts, H. J., van Ham, M., van Dam, P., Vuylsteke, P., Bastings, J., Kruitwagen, R. F. P. M., Lambrechts, S., Damink, S. W. M. O., Rensen, S. S., ... van Driel, W. J. (2020). No influence of sarcopenia on survival of ovarian cancer patients in a prospective validation study. Gynecologic Oncology, 159(3), 706-711. https://doi.org/10.1016/j.ygyno.2020.09.042

Document status and date:

Published: 01/12/2020

DOI:

10.1016/j.ygyno.2020.09.042

Document Version:

Publisher's PDF, also known as Version of record

Document license:

Taverne

Please check the document version of this publication:

- A submitted manuscript is the version of the article upon submission and before peer-review. There can be important differences between the submitted version and the official published version of record.

People interested in the research are advised to contact the author for the final version of the publication, or visit the DOI to the publisher's website.

- The final author version and the galley proof are versions of the publication after peer review.

- The final published version features the final layout of the paper including the volume, issue and page numbers.

Link to publication

\footnotetext{
General rights rights.

- You may freely distribute the URL identifying the publication in the public portal. please follow below link for the End User Agreement:

www.umlib.nl/taverne-license

Take down policy

If you believe that this document breaches copyright please contact us at:

repository@maastrichtuniversity.nl

providing details and we will investigate your claim.
}

Copyright and moral rights for the publications made accessible in the public portal are retained by the authors and/or other copyright owners and it is a condition of accessing publications that users recognise and abide by the legal requirements associated with these

- Users may download and print one copy of any publication from the public portal for the purpose of private study or research.

- You may not further distribute the material or use it for any profit-making activity or commercial gain

If the publication is distributed under the terms of Article $25 \mathrm{fa}$ of the Dutch Copyright Act, indicated by the "Taverne" license above, 


\title{
No influence of sarcopenia on survival of ovarian cancer patients in a prospective validation study
}

\author{
Jorne Ubachs ${ }^{\text {a,b,c,d,1 }}$, Simone N. Koole ${ }^{\text {ef, }, 1}$, Max Lahaye ${ }^{g}$, Cristina Fabris ${ }^{\text {g, }}{ }^{\text {, Leigh Bruijs }}{ }^{\text {e, }}$ \\ Jules Schagen van Leeuwen $^{\mathrm{h}}$, Henk W.R. Schreuder ${ }^{\mathrm{i}}$, R.H. Hermans ${ }^{\mathrm{j}}$, I.H. de Hingh ${ }^{\mathrm{k}}$, J. van der Velden ${ }^{1}$, \\ H.J. Arts ${ }^{\mathrm{m}}$, M. van Ham ${ }^{\mathrm{n}}$, P. van Dam ${ }^{\mathrm{o}}$, P. Vuylsteke ${ }^{\mathrm{p}}$, Jacco Bastings ${ }^{\mathrm{a}}$, Roy F.P.M. Kruitwagen ${ }^{\mathrm{a}, \mathrm{b}}$, \\ Sandrina Lambrechts ${ }^{\mathrm{a}, \mathrm{b}}$, Steven W.M. Olde Damink ${ }^{\mathrm{c}, \mathrm{d}, \mathrm{q}}$, Sander S. Rensen ${ }^{\mathrm{c}, \mathrm{d}}$, Toon Van Gorp ${ }^{\mathrm{r}}$, \\ Gabe S. Sonke ${ }^{\mathrm{f}}$, Willemien J. van Driel ${ }^{\mathrm{e}, *}$
}

a Department of Obstetrics and Gynecology, Maastricht University Medical Centre, Universiteitssingel 40, 6229ER Maastricht, the Netherlands

${ }^{\mathrm{b}}$ GROW - School for Oncology and Developmental Biology, Maastricht University, Universiteitssingel 40, 6229ER Maastricht, the Netherlands

c Department of Surgery, Maastricht University Medical Centre, Universiteitssingel 40, 6229ER Maastricht, the Netherlands

${ }^{\mathrm{d}}$ NUTRIM, school of Nutrition and Translational Research in Metabolism, Maastricht University, Universiteitssingel 40, 6229ER Maastricht, the

Netherlands

e Department of Gynecology, the Netherlands Cancer Institute, Plesmanlaan 121, 1066CX Amsterdam, the Netherlands

${ }^{\mathrm{f}}$ Department of Medical Oncology, the Netherlands Cancer Institute, Plesmanlaan 121, 1066CX Amsterdam, the Netherlands

g Department of Radiology, the Netherlands Cancer Institute, Plesmanlaan 121, 1066CX Amsterdam, the Netherlands

h Department of Obstetrics \& Gynecology, Sint Antonius Hospital, Koekoekslaan 1, 3435CM Nieuwegein, the Netherlands

${ }^{i}$ Department of Gynecological Oncology, UMC Utrecht Cancer Center, Heidelberglaan 100, 3584CX Utrecht, the Netherlands

${ }^{\mathrm{j}}$ Department of Gynecology and Obstetrics, Catharina Hospital, Michelangelolaan 2, 5623 EJ Eindhoven, the Netherlands

${ }^{\mathrm{k}}$ Department of Surgery, Catharina Hospital, Michelangelolaan 2, 5623 EJ Eindhoven, the Netherlands

${ }^{1}$ Department of Obstetrics and Gynecology, Academic Medical Center, Meibergdreef 9, 1105 AZ Amsterdam, the Netherlands

m Department of Gynecological Oncology, University Medical Center Groningen, Hanzeplein 1, 9713 GZ Groningen, the Netherlands

n Department of Gynecological oncology, Radboud University Medical Center, Geert Grooteplein Zuid 10, 6525 GA Nijmegen, the Netherlands

${ }^{\circ}$ Department of Gynecologic Oncology, University Hospital Antwerp, Wilrijkstraat 10, 2650 Antwerp, Belgium

p Department of Medical Oncology, UCLouvain and University of Botswana, CHU Ucl Namur, Belgium

${ }^{\mathrm{q}}$ Department of Visceral- and Transplantation Surgery, RWTH Aachen University, Templergraben 55, 52062 Aachen, Germany

${ }^{\mathrm{r}}$ Department of Obstetrics and Gynecology, Division of Gynecological Oncology, University Hospitals Leuven, Leuven Cancer Institute, Herestraat 49 , 3000 Leuven, Belgium

\section{H I G H L I G H T S}

- Longitudinal CT-scans were assessed for body composition alterations during neoadjuvant chemotherapy (NACT).

- A decrease in skeletal muscle index during NACT was not predictive for recurrence-free survival or overall survival.

- A decrease in skeletal muscle index was associated with more pre-treatment and pre-operative reported adverse events.

\section{A R T I C L E I N F O}

\section{Article history:}

Received 8 May 2020

Accepted 26 September 2020

Available online 2 October 2020

\section{Keywords:}

Sarcopenia

Ovarian cancer

Cachexia

Survival

\section{A B S T R A C T}

Objective. Decrease in skeletal muscle index (SMI) during neoadjuvant chemotherapy (NACT) has been associated with worse outcome in patients with advanced ovarian cancer. To validate these findings, we tested if a decrease in SMI was a prognostic factor for a homogenous cohort of patients who received NACT in the randomized phase 3 OVHIPEC-trial.

Methods. CT-scans were performed at baseline and after two cycles of neoadjuvant chemotherapy in stage III ovarian cancer patients. The SMI ( skeletal muscle area in $\mathrm{cm}^{2}$ divided by body surface area in $\mathrm{m}^{2}$ ) was calculated using SliceOMatic software. The difference in SMI between both CT-scans ( $\Delta$ SMI) was calculated. Cox-regression analyses were performed to analyze the independent effect of a difference in SMI ( $\Delta \mathrm{SMI})$ on outcome. Log-rank tests were performed to plot recurrence-free (RFS) and overall survival (OS). The mean number of adverse events per patient were compared between groups using $t$-tests.

\footnotetext{
* Corresponding author at: Department of Gynecology, the Netherlands Cancer Institute, Plesmanlaan 121, 1006BE Amsterdam, the Netherlands.

E-mail address: w.v.driel@nki.nl (W.J. van Driel).

1 These authors equally contributed to this article.

2 Present address: Department of Radiology, University of Verona Hospital, Piazzale Aristide Stefani 1, 37126 Verona, Italy.
} 

the group with a decrease in $\triangle$ SMI and 73 of 138 of the patients (53\%) in the group with stable/increase in $\Delta$ SMI had died. Median RFS and OS did not differ significantly ( $p=0.297$ and $p=0.764)$ between groups. Patients with a decrease in SMI experienced more pre-operative adverse events, and more grade 3-4 adverse events.

Conclusion. Decreased SMI during neoadjuvant chemotherapy was not associated with worse outcome in patients with stage III ovarian cancer included in the OVHIPEC-trial. However, a strong association between decreasing SMI and adverse events was found.

\section{Introduction}

Epithelial ovarian cancer shows the highest mortality rate of all gynaecologic cancers in the western world [1]. The majority of patients is diagnosed with advanced stage disease, (FIGO $\geq$ III) [2]. For advanced stage patients, 10 -year survival is only $10-15 \%$, and long term survival has not improved over the past 20 years [3]. Standard treatment consists of maximum cytoreductive surgery (CRS) combined with chemotherapy consisting of carboplatin and paclitaxel. If complete primary CRS is not feasible, interval CRS might be performed after three cycles of neoadjuvant chemotherapy [4-6]. Achieving a complete cytoreduction is the most important prognostic factor, in addition to the ability to complete six cycles of carboplatin and paclitaxel and the clinical condition of the patient $[7,8]$. The clinical condition is reflected by multiple factors and besides subjective scores, such as the World Health Organization (WHO) performance score, we lack an objective measure of patient fitness. Sarcopenia might be such a measure, and might be predictive for treatment completion and outcome in patients with advanced stage ovarian cancer.

Sarcopenia is the loss of, or low, skeletal muscle mass [9]. The skeletal muscle mass of patients with cancer can be accurately quantified using a single lumbar CT image by the so-called skeletal muscle index (SMI) [10]. Loss of skeletal muscle mass is associated with higher rates of chemotoxicity and impaired overall survival (OS) in ovarian cancer patients [11-13]. In an analysis of a retrospective cohort of 123 patients published by Rutten et al., loss of skeletal muscle mass during NACT was shown to be associated with worse outcome. However, multivariate analyses taking into account completeness of surgery, response to chemotherapy, and toxicity were not performed [13]. Although previous studies reported worse outcome in patients with ovarian cancer and either a low baseline SMI or a decrease in SMI during treatment, it is uncertain whether SMI is an independent prognostic factor or a measure of extensive disease and treatment burden and poor performance [14-16].

The aim of this study was to validate earlier results by Rutten et al. in the most homogenous cohort of ovarian cancer patients reported to date. We analyzed whether a decrease in SMI during NACT was associated with worse outcome in a cohort of patients with similar extent of disease; stage III epithelial ovarian cancer, and similar performance scores.

\section{Patients and methods}

\subsection{Patients and treatment}

For this study, CT-scans were collected from patients included in the OVHIPEC trial [17]. This multicenter randomized phase III trial included 245 newly-diagnosed patients with stage III epithelial ovarian, fallopian tube, or peritoneal cancer between April 2007 and April 2016. Full eligibility criteria are presented elsewhere [17]. All patients received three cycles of intravenous chemotherapy (carboplatin [area under the curve $5-6 \mathrm{mg} / \mathrm{ml}$ per minute] and paclitaxel [175 $\left.\mathrm{mg} / \mathrm{m}^{2}\right]$ ) prior to interval cytoreductive surgery, because of extent of disease, or because of incomplete primary CRS (residual tumor $>1 \mathrm{~cm}$ in diameter). Patients were eligible for inclusion in case of at least stable disease after two cycles of NACT. Randomization was performed during interval cytoreductive surgery when complete or optimal (no visible, or $<1 \mathrm{~cm}$ visible tumor remaining) CRS was anticipated. Patients were randomized to receive either interval CRS with or without HIPEC using cisplatin $100 \mathrm{mg} / \mathrm{m}^{2}$. In both groups disease was too extensive for primary surgery, either defined by incomplete primary surgery or based on radiological imaging. Ten percent of patients included in the OVHIPEC-trail were primarily treated with incomplete primary cytoreduction before administration of chemotherapy and complete or near-complete interval cytoreduction, numbers were equally distributed between arms due to randomization. Subgroup analysis in the OVHIPEC study showed no difference in outcome between patients treated with initial incomplete surgery and those who started with neoadjuvant chemotherapy. An additional three cycles of adjuvant intravenous carboplatin were administered after CRS for all patients. CT-scans were performed at diagnosis: before start of neoadjuvant chemotherapy (NACT), after 2 cycles of NACT, at the end of adjuvant chemotherapy, and at 6,12 , and 24 months after the last cycle of chemotherapy. Grade $1-5$ toxicity was scored from baseline to 30 days after end of therapy, using the National Cancer Institute Common Terminology Criteria for Adverse Events (CTCAE) version 4.0. All patients provided informed consent for participation. The trial protocol was approved by the institutional review board of the Netherlands Cancer Institute. The trial was registered in the international database: ClinicalTrials.gov Identifier: NCT00426257.

\subsection{Body composition analysis}

To analyze whether pre-operative sarcopenia and/or a skeletal muscle depletion were associated with worse outcome, CT-scans performed at baseline (timepoint 1 ) and after 2 cycles of neoadjuvant chemotherapy (timepoint 2) were selected. The axial slide at the third lumbar level (L3), with the best representation of the bilateral transverse process was selected from each portal venous phase CT-scan.

The axial CT-scan at the L3 level was used for evaluation of total skeletal muscle (SM). Predefined and previously validated cut-offs of -29 to +150 Hounsfield Units (HU) [18] for SM were used to demarcate tissue using SliceOmatic software (v5.0, Tomovision, Montreal, Canada). Evaluation of the demarcations was performed by one of two observers (JU \& JB), both blinded for clinical characteristics, outcome and allocated treatment arm. An example of how tissues were delineated with SliceOmatic is shown in Fig. 1. The Skeletal Muscle Index (SMI) was calculated using the surface area of SM in $\mathrm{cm}^{2}$ and the stature of the patient (squared height) in $\mathrm{m}^{2}$. All data were coded and processed anonymously.

Changes in SMI from timepoint 1 to timepoint 2 were expressed as a percentage. To account for variations in time between the sequential CT-scans, the change in SMI was calculated as a percentage of change per 100 days. In order to do so, the change in SMI between the scan at timepoint 1 and timepoint 2 was divided by the number of days between the scans, and subsequently multiplied by 100 . This is referred to as $\triangle$ SMI. A measurement error of $2 \%$ was adopted based on previously reported accuracy of CT for muscle and fat tissue analysis [10]. Patients with a decrease of $>2 \%$ of SMI in 100 days were defined as the SMI-loss group. The $2 \%$ cutoff was also used by Rutten et al. [13]. Other patients were defined as SMI-stable or increase [10,13]. 


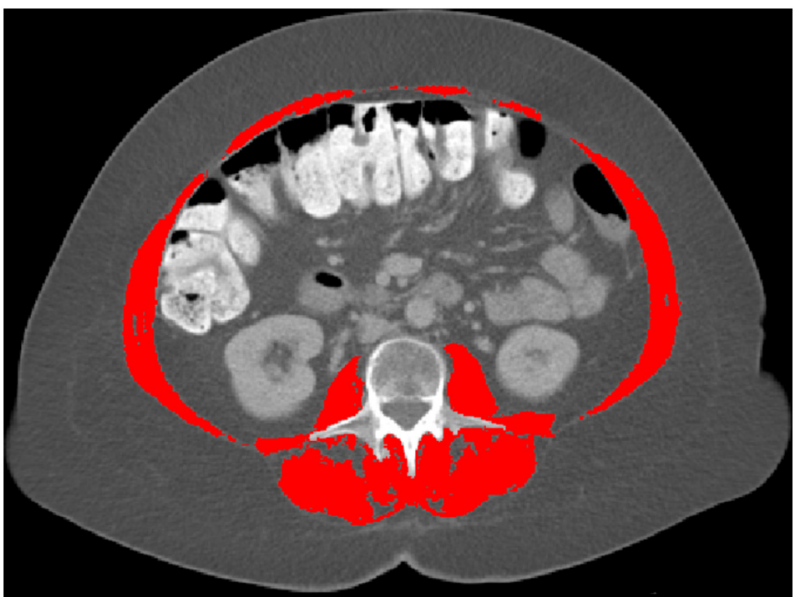

Fig. 1. Abdominal axial CT scan of an ovarian cancer patient taken at the third lumbar level. Total skeletal muscle area in red.

Only CT-scans that were performed up to 10 weeks before start of chemotherapy, up to interval CRS surgery were selected. Scans at timepoint 1 had to be performed ten weeks to 1 day before start of neoadjuvant chemotherapy. Scans at timepoint 2 had to be performed more than three weeks after start of neoadjuvant chemotherapy but before interval CRS.

\subsection{Statistical analyses}

Comparisons between the SMI-loss and SMI-stable/increase group were performed with the t-test for continuous variables and the Pearson's chi-square test for categorical variables. Pre-operative toxicity was scored before start of chemotherapy, during NACT, or between NACT and surgery. Toxicity was divided into CTCAE grade 1-2 or grade 3-5 toxicity. The mean number of events per patient were presented and compared between groups using a t-test for independent samples. The Welch t-test was used to compare the total number of pre-operative adverse events.

Survival was compared for sarcopenic patients at timepoint 1 and timepoint 2, and for the SMI-loss group and the SMI-stable or increase group. Analyses of overall survival (OS) and recurrence-free survival (RFS) were performed using Kaplan-Meier estimates and log-rank tests. RFS was defined as the time from randomization to firstrecurrence or death, whichever occurred first, as was defined by the GCIG criteria [19]. Univariate and multivariable cox regression analyses was performed for analyzing the effect of the different treatment arms on outcome, with deltaSMI and treatment arm (HIPEC or no HIPEC) as associated variables. Subgroup analyses were performed for interval CRS and interval CRS + HIPEC groups for OS and RFS. Statistical significance for all comparisons was determined at $p<0.05$. All analyses were performed with the statistical software package SPSS v.25.0 (IBM Corp, Chicago, IL, USA).

\section{Results}

Paired CT-scans at baseline and after two cycles of NACT were available for 221 out of 245 patients (90.2\%) For 24 patients, CT-scans were not provided by the participating centers. Mean time between CT-scan 1 and CT-scan 2 was 60 days (SD 15.7). Mean time between cycles of NACT and interval CRS are presented in supplementary table S1. The SMI and $\Delta$ SMI could not be calculated for nine patients (4.1\%) because CT-scans were of insufficient quality or incomplete. The median SMI was 39.5 (range 27.5-57.9) at timepoint 1 and 38.1 (range 26.4-55.4) at timepoint 2. Means with SD of the SMI at timepoint 1 and 2, and
$\Delta$ SMI are presented in supplementary table S2. The $\Delta$ SMI could be calculated for 212 patients. The mean $\Delta$ SMI was $-5.9 \%$ (SD 11.8\%), ranging from $-31.6 \%$ to $46.9 \%$. $\Delta$ SMI was lower than $-2 \%$ in 138 patients and was stable or increased (higher than $-2 \%$ ) in 74 patients. Baseline characteristics for all patients with SMI measurements are presented in Tables 1 and 2. The mean BMI in the SMI stable/ increase group was 20.9 (SD 3.6), and 19.7 (SD 3.1) in the SMI decrease group ( $p=$ 0.025 ). Slightly more patients with a decrease in SMI were treated with HIPEC ( $n=74(53.6 \%)$ ), compared to the group with stable/increased SMI $(n=29$ (39.2\%) $p=0.045)$. Median OS was 41 months (95\% confidence interval [CI] 36.1-45.9), and median RFS was 12 months (95\% CI 10.6-13.4).

\subsection{Toxicity and sarcopenia}

Between enrollment and surgery, pre-operative toxicity was scored. A total of 1265 events were registered before interval cytoreductive surgery. The majority of the events was a CTCAE grade 1-2 event (1208/ 1265; 95.5\%) (Table 3). Within the group of patients with a decrease in SMI, a total of 893 events (70.6\%) were reported, compared to 372 (29.4\%) events in the stable/increase SMI group $(p=0.008)$. The overall mean number of pre-operative events per patient was 6.2 (SD 3.9). The percentage of grade 3-4 events was higher in the group with a decrease in SMI (5.3\%) than the percentage in the group with stable or increased SMI (2.6\%). The mean number of pre-operative events was significantly higher: 6.7 (SD 3.9) in the group of patients whose SMI decreased versus 5.3 (SD 3.1) in the group with a stable or increased SMI ( $p=$ 0.019 ) (Table 3). Specific grade 3-4 toxicities are listed in supplementary table S5, and were mostly related to chemotherapy or cancerrelated adverse events such as pulmonary embolisms.

\subsection{Sarcopenia and survival}

After a median of 4.7 years of follow-up, 116 of 212 patients (55\%) had died and 181 of 212 patients (85\%) experienced an event of disease-recurrence or death. Survival analysis revealed that 43 of 74 patients (58\%) with a more than $2 \%$ decrease in SMI versus 73 of 138 (53\%) of the patients with a stable or increased SMI had died. Median overall survival did not differ significantly between these two groups ( $p=$ 0.764 , Fig. 2). 61 of $74(82 \%)$ patients in the $\Delta$ SMI decrease group had disease recurrence, compared to 120 of 138 (87\%) in the $\Delta$ SMI stable/increase group. Median recurrence-free survival was similar $(p=0.297$, Fig. 3). $\Delta$ SMI was also not associated with overall survival and recurrence-free survival after correcting for treatment effect of HIPEC in multivariate analysis (supplementary tables S3 and S4, respectively).

\section{Discussion}

The primary goal of this study was to validate the previously published finding that a decline in SMI during NACT in ovarian cancer patients was associated with worse OS [13]. Although a loss of skeletal muscle mass was detected in the present cohort, inclusion criteria allowed only for randomization of patients with prognostically favorable characteristics such as good response to chemotherapy or complete- or optimal cytoreduction. This selection of patients might have resulted in negative validation of the results as published by Rutten et al. Nevertheless, a strong association between decreasing SMI and adverse events was found.

Rutten et al. showed that ovarian cancer patients with a decrease in SMI ( $>2 \% / 100$ days) had a significant $(p=0.004)$ survival disadvantage (median OS $916 \pm 99$ days) as opposed to patients who showed stable or increased SMI (median OS $1431 \pm 470$ days) [13]. The patient cohort presented by Rutten et al. is essentially different regarding a number of aspects: 1) patients were older (mean age 60.9 [present] vs. 66.5 [Rutten et al.]), 2) had higher FIGO stage (III [present] vs. III-IV [Rutten et al.]), 3) received more chemotherapy between CT-scans (2 [present] 
Table 1

Patient and treatment characteristics.

\begin{tabular}{|c|c|c|c|c|}
\hline & $\begin{array}{l}\text { Total cohort } \\
N=212\end{array}$ & $\begin{array}{l}\text { SMI decrease }>2 \% \\
N=138\end{array}$ & $\begin{array}{l}\text { SMI stable or SMI increase } \\
N=74\end{array}$ & $P$-value \\
\hline Age, mean years (SD) & $60.9(8.1)$ & $60.9(8.2)$ & $61.0(7.8)$ & 0.883 \\
\hline \multicolumn{5}{|l|}{ FIGO, Nr. (\%) } \\
\hline III & $221(100)$ & $138(100)$ & $74(100)$ & \\
\hline Histological type (\%) & & & & 0.469 \\
\hline High-grade serous & $191(90.1)$ & $125(90.6)$ & $66(89.2)$ & \\
\hline Other & $19(9.0)$ & $11(8.0)$ & $8(10.8)$ & \\
\hline Unknown & $2(0.9)$ & $2(1.4)$ & 0 & \\
\hline BMI, mean $\mathrm{kg} / \mathrm{m}^{2}(\mathrm{SD})^{*}$ & & & & 0.025 \\
\hline Baseline & $20.5(3.5)$ & $20.9(3.6)$ & $19.7(3.1)$ & \\
\hline Weight, mean kg (SD)* & & & & 0.076 \\
\hline Baseline & $68.9(12.9)$ & $70.1(13.2)$ & $66.8(12.2)$ & \\
\hline Primary incomplete debulking, Nr. (\%) & & & & 0.884 \\
\hline yes & $18(8.5)$ & $12(8.7)$ & $6(8.1)$ & \\
\hline no & $194(91.5)$ & $126(91.3)$ & $68(91.9)$ & \\
\hline Outcome interval CRS, Nr. (\%) $\dagger$ & & & & 0.936 \\
\hline $\mathrm{R} 1$ & $146(68.9)$ & $95(68.8)$ & $51(68.9)$ & \\
\hline $\mathrm{R} 2 \mathrm{a}$ & $40(18.9)$ & $26(18.8)$ & $14(18.9)$ & \\
\hline $\mathrm{R} 2 \mathrm{~b}$ & $22(10.4)$ & $14(10.1)$ & $8(10.8)$ & \\
\hline Suboptimal & $1(0.5)$ & $1(0.7)$ & 0 & \\
\hline No resection & $1(0.5)$ & $1(0.7)$ & 0 & \\
\hline No surgery & $2(0.9)$ & $1(0.7)$ & $1(1.4)$ & \\
\hline Treatment with HIPEC, Nr (\%) & & & & 0.045 \\
\hline HIPEC & $103(48.6)$ & $74(53.6)$ & $29(39.2)$ & \\
\hline No HIPEC & $109(51.4)$ & $64(46.4)$ & $45(60.8)$ & \\
\hline Six cycles of chemotherapy completed, $\mathrm{Nr}$. (\%) & & & & 0.895 \\
\hline Yes & $197(92.9)$ & $128(92.8)$ & $69(93.2)$ & \\
\hline No & $15(7.1)$ & $10(7.2)$ & $5(6.8)$ & \\
\hline
\end{tabular}

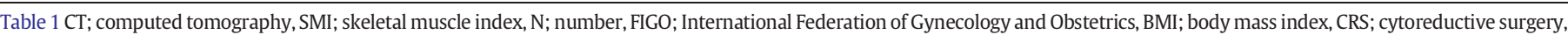
NA; not applicable, HIPEC; hyperthermic intraperitoneal chemotherapy, $\mathrm{Nr}$; number.

* based on known values.

+ R1: no visible tumor, complete cytoreduction; R2a: tumor nodules $\leq 2.5 \mathrm{~mm}$; R2b: tumor nodules $>2.5 \mathrm{~mm}$ and $\leq 10 \mathrm{~mm}$; suboptimal: tumor nodules $>10$ mm, incomplete cytoreduction.

vs. 3 cycles [Rutten et al.]), and 4) had worse outcome of CRS (68\% [present] vs. $45.5 \%$ [Rutten et al.] patients with complete debulking surgery). The present study included patients under the age of 76, who were fit for major surgery (ASA1-2 and WHO performance score $0-2$ ), had adequate organ function, and had only FIGO stage III disease [17]. In addition, only patients with a complete or partial response, or stable disease after two cycles, were selected. However, the mean decrease of SMI in both cohorts was quite similar (5.9\%/100 days present cohort vs. $5.2 \% / 100$ days in the Rutten et al. cohort).
During NACT, patients with a decrease in SMI had a significantly higher number of toxicities of any grade (6.7, SD: 4.2 vs. 5.3 ; SD: 3.1 , $p=0.008$ ) (Table 3 ). This might indicate that skeletal muscle depletion is a measure of clinical fitness which impacts the patients' ability to receive treatment and thereby affects survival, rather than being an independent prognostic variable. Earlier findings already indicated that increased toxicity from chemotherapy is related to lower lean body mass, which results in reduced volume of distribution, protein binding, metabolism, and clearance of drugs [20]. The group of patients who lost

Table 2

CT-scans and body composition characteristics.

\begin{tabular}{|c|c|c|c|c|}
\hline & $\begin{array}{l}\text { Total cohort } \\
\mathrm{N}=212\end{array}$ & $\begin{array}{l}\text { SMI decrease }>2 \% \\
\mathrm{~N}=138\end{array}$ & $\begin{array}{l}\text { SMI stable or SMI increase } \\
\mathrm{N}=74\end{array}$ & P-value \\
\hline \multicolumn{5}{|c|}{ Nr. of days between scan 1-2, mean (SD) } \\
\hline & $60.3(15.7)$ & $60.1(16.0)$ & $60.6(15.3)$ & 0.841 \\
\hline \multicolumn{5}{|c|}{ Nr. of days between start NACT and scan 2 , mean (SD)* } \\
\hline \multirow{2}{*}{\multicolumn{5}{|c|}{$\mathrm{SMI}$, mean $\mathrm{cm}^{2} / \mathrm{m}^{2}(\mathrm{SD})$}} \\
\hline & & & & \\
\hline Baseline & $39.5(5.4)$ & $40.7(5.3)$ & $37.4(4.0)$ & $<0.001$ \\
\hline After 2 cycles of NACT & $38.1(5.0)$ & $37.8(5.0)$ & $38.6(5.0)$ & 0.252 \\
\hline$\%$ of change (range) & $-5.9 \%(-31.6 \%$ to $46.9 \%)$ & $-12.3(-31.6 \%$ to $-2.3 \%)$ & $6.0 \%(-2.0 \%-46.9 \%)$ & $<0.001$ \\
\hline \multicolumn{5}{|l|}{ SMRA, mean HU (SD)* } \\
\hline Baseline & $36.0(7.8)$ & $35.7(7.6)$ & $36.5(8.2)$ & 0.464 \\
\hline After 2 cycles of NACT & $36.6(7.5)$ & $35.6(7.2)$ & $38.5(7.7)$ & 0.007 \\
\hline $\begin{array}{l}\text { OS, median months } \\
\quad(95 \% \mathrm{CI})\end{array}$ & $41(36.11-45.89)$ & $41(34.18-47.82)$ & $41(35.54-46.46)$ & 0.764 \\
\hline $\begin{array}{l}\text { RFS, median months } \\
\quad(95 \% \mathrm{CI})\end{array}$ & $12(10.63-13.37)$ & $11(9.49-12.51)$ & $13(10.24-15.76)$ & 0.297 \\
\hline
\end{tabular}

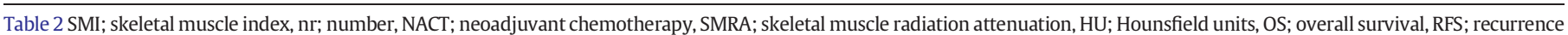
free survival, 95\% CI; 95\% confidence interval.

* based on known values. 
Table 3

Association of decrease in SMI with adverse events.

\begin{tabular}{|c|c|c|c|c|}
\hline & $\begin{array}{l}\text { Total } \\
\text { cohort } \\
N=203\end{array}$ & $\begin{array}{l}\text { SMI } \\
\text { decrease }>2 \% \\
N=133\end{array}$ & $\begin{array}{l}\text { SMI stable } \\
\text { or SMI increase } \\
N=70\end{array}$ & P-value \\
\hline \multicolumn{5}{|c|}{ Mean number of adverse events pre-operative (SD)* } \\
\hline Gr1-2 & $5.9(3.7)$ & $6.4(3.9)$ & $5.2(3.1)$ & 0.044 \\
\hline Gr3-4 & $0.3(0.7)$ & $0.4(0.8)$ & $0.1(0.4)$ & $<0.001$ \\
\hline $\begin{array}{l}\text { Mean } \mathrm{N} \text { of events } \\
\text { pre-op per pt }\end{array}$ & $6.2(3.9)$ & $6.7(4.2)$ & $5.3(3.1)$ & 0.019 \\
\hline \multicolumn{5}{|c|}{ Total number of adverse events pre-operative* } \\
\hline $\begin{array}{l}\text { Total Gr1-2 } \\
\quad \text { (range per patient) }\end{array}$ & $1208(0-22)$ & $846(0-22)$ & $362(1-13)$ & 0.018 \\
\hline $\begin{array}{l}\text { Total Gr3-4 } \\
\quad \text { (range per patient) }\end{array}$ & $57(0-4)$ & $47(0-4)$ & $10(0-2)$ & 0.016 \\
\hline $\begin{array}{l}\text { Total nr of events } \\
\quad \text { (range per patient) }\end{array}$ & $1265(1-25)$ & $893(1-25)$ & $372(1-13)$ & 0.008 \\
\hline
\end{tabular}

CT; computed tomography, SMI; skeletal muscle index, SD; standard deviation, Gr; grade, $\mathrm{N}$; number.

* based on known values.

skeletal muscle during chemotherapy had a slightly higher baseline BMI (20.9, SD 3.6) compared to the group of patients with stable/increased muscle mass (19.7, SD 3.1). It is not clear whether this slightly higher baseline BMI contributes to a greater decrease in skeletal muscle mass [21].

The association between a decrease in SMI and outcome that was previously described [13] may be attributed to the fact that relatively frail patients were studied that did not meet the inclusion criteria for the OVHIPEC trial, but had a poor outcome due to adverse events, dose modifications or incomplete surgery. The strong relationship with reported toxicities in the current study is in line with this explanation. One of the main shortcomings of the current survival analysis is that it was not powered for analysis of OS/RFS, or for any subgroup analyses. All participants in the OVHIPEC trial were included for analysis with the assumption that sarcopenic patients were evenly distributed over both treatment arms. However, slightly more patients were treated with HIPEC in the group with a decrease in SMI. Multivariate analyses for treatment arm (HIPEC vs. no HIPEC) and for delta SMI were performed to evaluate the independent effect of deltaSMI without the potential mask by treatment with HIPEC (supplementary table S3 and S4). Skeletal muscle mass was not an independent prognostic factor

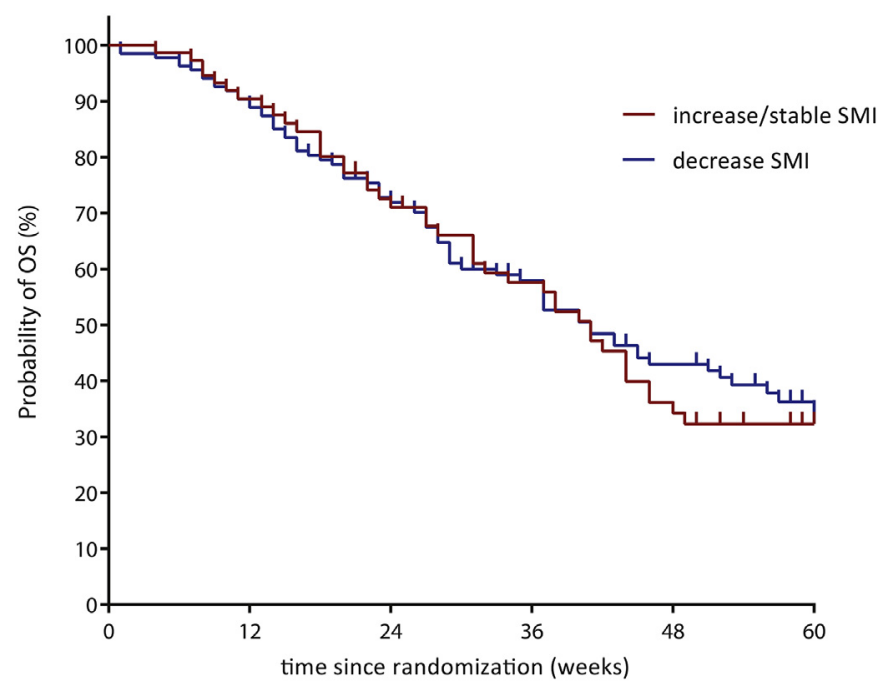

Fig. 2. Kaplan-Meier survival analysis. SMI; skeletal muscle index, plotted is an increase vs. decrease in SMI and the association with overall survival.

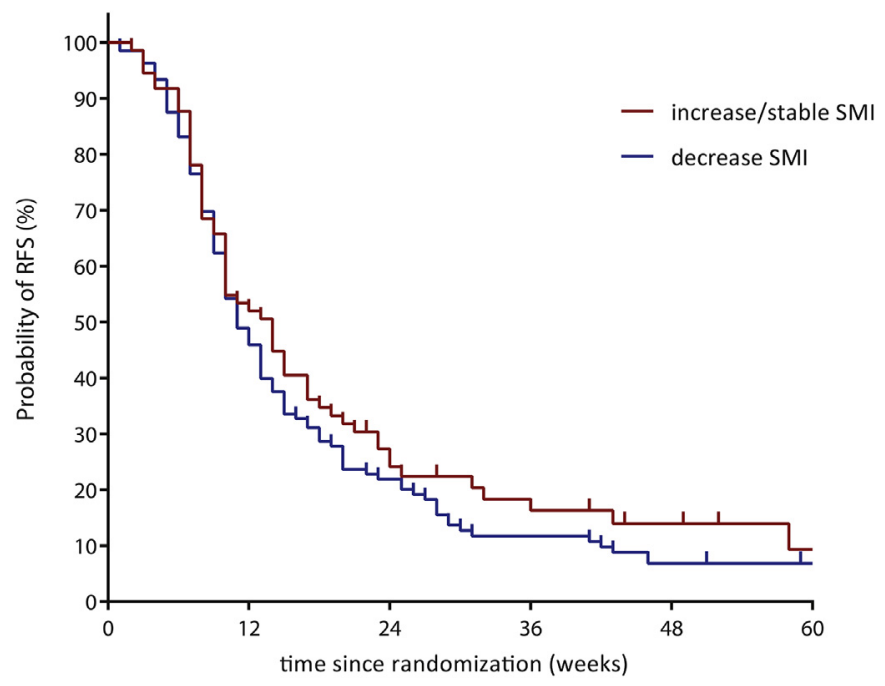

Fig. 3. Kaplan-Meier survival analysis. SMI; skeletal muscle index, plotted is an increase vs. decrease in SMI and the association with recurrence free survival.

for recurrence-free and overall survival in multivariate analysis. Due to its prospective nature, this cohort is the most homogeneous described to date, and important confounders for outcome (FIGO stage, performance score, and surgical treatment) which were present in previous studies have been eliminated. The strict inclusion criteria in the current study make it difficult to generalize our findings to the general ovarian cancer patient population. Assessment of SMI was performed according to accepted methods $[10,22,23]$. The effect of a decrease in SMI was assessed independently of treatment with HIPEC in multivariate analysis. Since a survival disadvantage was not detected for patients with skeletal muscle mass loss, it is expected that optimal resection of the tumor is of greater importance for OS than adverse body composition. Based on previous cohorts, the association between adverse body composition and OS might still be of importance in older patients, or patients in whom complete resection of the tumor is not feasible. Due to the nature of the selected cohort, however, these patients were not analyzed $[14,15,24,25]$. Multivariate analyses which take into account important prognostic variables such as treatment response, completeness of surgery, and toxicities could reveal if a decrease in body composition might be an independent predictor in other cohorts.

\section{Conclusion}

A change in SMI during 2 cycles of NACT was not associated with outcome within a large cohort of patients included in the OVHIPEC trial. In the current population, with relatively good prognostic characteristics, a loss of skeletal muscle mass is not an independent predictor for survival. In this selection of patients, we observed a significant association of sarcopenia with reported toxicities. Whether loss of skeletal muscle mass is related to patient performance status, toxicity or treatment burden should be confirmed in future analyses.

\section{Author contributions}

Drs. Ubachs and Koole wrote the initial drafts of the manuscript and performed the analyses. Dr. Lahaye and Fabris a responsible for CT-scan observations. Drs. Bruijs, Koole and Ubachs were responsible for data collection. Dr. Schagen van Leeuwen, Schreuder, Hermans, de Hingh, van der Velden, Arts, and van Driel are responsible for patient acquisition and data collection. Dr. Bastings, Kruitwagen, Lambrechts, Olde Damink, Rensen, van Gorp, Sonke and van Driel contributed clinical 
expertise to the analyses and manuscript. All authors reviewed the manuscript.

\section{Research support}

The original trial was funded by the Dutch Cancer Society;

ClinicalTrials.gov number, NCT00426257

\section{Declaration of Competing Interest}

Dr. Sonke reports institutional research support outside the submitted work, from AstraZeneca, Merck, Novartis, and Roche, during the conduct of the study.

Dr. Dam reports research support outside the submitted work from, Roche, Novartis, and Amgen. Travel and speakers' fees were received from Roche, Novartis, and Teva.

Dr. De Hingh reports grants from ROCHE, and grants from QP\&S/ RanD, outside the submitted work.

Dr. Vuylsteke reports advisory board fees, travel grants, and speaker fees from MSD, BMS, Pfizer, Novartis, Lilly, and Roche, outside the submitted work.

The other authors have no conflicts of interest to declare.

\section{Appendix A. Supplementary data}

Supplementary data to this article can be found online at https://doi. org/10.1016/j.ygyno.2020.09.042.

\section{References}

[1] A.P. Heintz, et al., Carcinoma of the ovary. FIGO 26th annual report on the results of treatment in gynecological cancer, Int. J. Gynaecol. Obstet. 95 (Suppl. 1) (2006) S161-S192.

[2] S. Narod, Can advanced-stage ovarian cancer be cured? Nat. Rev. Clin. Oncol. 13 (4) (2016) 255-261.

[3] M. Timmermans, et al., No improvement in long-term survival for epithelial ovarian cancer patients: a population-based study between 1989 and 2014 in the Netherlands, Eur. J. Cancer 88 (2017) 31-37.

[4] M.E. van der Burg, et al., The effect of debulking surgery after induction chemotherapy on the prognosis in advanced epithelial ovarian cancer. Gynecological Cancer cooperative Group of the European Organization for research and treatment of Cancer, N. Engl. J. Med. 332 (10) (1995) 629-634.

[5] I. Vergote, et al., Neoadjuvant chemotherapy or primary surgery in stage IIIC or IV ovarian cancer, N. Engl. J. Med. 363 (10) (2010) 943-953.
[6] A.A. Wright, et al., Neoadjuvant chemotherapy for newly diagnosed, advanced ovarian cancer: Society of Gynecologic Oncology and American Society of clinical oncology clinical practice guideline, Gynecol. Oncol. 143 (1) (2016) 3-15.

[7] S.J. Chang, et al., Survival impact of complete cytoreduction to no gross residual disease for advanced-stage ovarian cancer: a meta-analysis, Gynecol. Oncol. 130 (3) (2013) 493-498.

[8] M. Timmermans, et al., Interval between debulking surgery and adjuvant chemotherapy is associated with overall survival in patients with advanced ovarian cancer, Gynecol. Oncol. 150 (3) (2018) 446-450.

[9] K. Fearon, et al., Definition and classification of cancer cachexia: an international consensus, Lancet Oncol. 12 (5) (2011) 489-495.

[10] M. Mourtzakis, et al., A practical and precise approach to quantification of body composition in cancer patients using computed tomography images acquired during routine care, Appl. Physiol. Nutr. Metab. 33 (5) (2008) 997-1006.

[11] C.M. Prado, et al., The association between body composition and toxicities from the combination of Doxil and trabectedin in patients with advanced relapsed ovarian cancer, Appl. Physiol. Nutr. Metab. 39 (6) (2014) 693-698.

[12] T. Yoshikawa, et al., Psoas muscle volume as a predictor of peripheral neurotoxicity induced by primary chemotherapy in ovarian cancers, Cancer Chemother. Pharmacol. 80 (3) (2017) 555-561.

[13] I.J. Rutten, et al., Loss of skeletal muscle during neoadjuvant chemotherapy is related to decreased survival in ovarian cancer patients, J. Cachexia. Sarcopenia Muscle 7 (4) (2016) 458-466.

[14] I.J. Rutten, et al., The influence of sarcopenia on survival and surgical complications in ovarian cancer patients undergoing primary debulking surgery, Eur. J. Surg. Oncol. 43 (4) (2017) 717-724.

[15] H. Bronger, et al., Sarcopenia in advanced serous ovarian Cancer, Int. J. Gynecol. Cancer 27 (2) (2017) 223-232.

[16] N. Silva de Paula, et al., Sarcopenia and skeletal muscle quality as predictors of postoperative complication and early mortality in gynecologic cancer, Int. J. Gynecol. Cancer 28 (2) (2018) 412-420.

[17] W.J. van Driel, et al., Hyperthermic intraperitoneal chemotherapy in ovarian cancer N. Engl. J. Med. 378 (3) (2018) 230-240.

[18] N. Mitsiopoulos, et al., Cadaver validation of skeletal muscle measurement by magnetic resonance imaging and computerized tomography, J. Appl. Physiol. 85 (1) (1985) 115-122 1998

[19] G.J. Rustin, et al., Definitions for response and progression in ovarian cancer clinical trials incorporating RECIST 1.1 and CA 125 agreed by the gynecological cancer intergroup (GCIG), Int. J. Gynecol. Cancer 21 (2) (2011) 419-423.

[20] J.J. Hopkins, M.B. Sawyer, A review of body composition and pharmacokinetics in oncology, Expert. Rev. Clin. Pharmacol. 10 (9) (2017) 947-956.

[21] L. Martin, et al., Cancer cachexia in the age of obesity: skeletal muscle depletion is a powerful prognostic factor, independent of body mass index, J. Clin. Oncol. 31 (12) (2013) 1539-1547.

[22] I.J.G. Rutten, et al., Psoas muscle area is not representative of total skeletal muscle area in the assessment of sarcopenia in ovarian cancer, J. Cachexia. Sarcopenia Muscle 8 (4) (2017) 630-638.

[23] V.E. Baracos, Psoas as a sentinel muscle for sarcopenia: a flawed premise, J. Cachexia. Sarcopenia Muscle 8 (4) (2017) 527-528.

[24] A. Kumar, et al., Muscle composition measured by CT scan is a measurable predictor of overall survival in advanced ovarian cancer, Gynecol. Oncol. 142 (2) (2016) 311-316.

[25] B. Ataseven, et al., Skeletal muscle attenuation (sarcopenia) predicts reduced overal survival in patients with advanced epithelial ovarian Cancer undergoing primary Debulking surgery, Ann. Surg. Oncol. 25 (11) (2018) 3372-3379. 